\title{
Hyperbaric oxygen therapy as adjuvant therapy for odontogenic necrotizing myositis: case report
}

\author{
Andrea Neville Cracchiolo ${ }^{1}$, Daniela Maria Palma ${ }^{1}$, Marco Palmeri ${ }^{1}$, Fabio Genco ${ }^{1}$, Rosalia \\ Lo Bue ${ }^{1}$, Diego Tantillo ${ }^{1}$, Andrea Braconi ${ }^{1}$, Claudio Caramanna ${ }^{1}$, and Luigi Solazzo ${ }^{1}$ \\ ${ }^{1}$ Azienda Ospedaliera di Rilievo Nazionale e di Alta Specializzazione Civico Di Cristina \\ Benfratelli
}

April 14, 2021

\begin{abstract}
Necrotizing soft tissue infections (NSTIs) are life-threatening conditions burdened with high mortality. They're often associated to a poor prognosis if early diagnosis and prompt treatment is not warranted. Treatment is always medical, often surgical and sometimes with hyperbaric oxygen.
\end{abstract}

\section{Introduction}

Necrotizing soft tissue infections (NSTIs) are life-threatening conditions firstly described by Wilson in 1952. He portrayed a rare infection characterized by bacteria-induced necrosis of the subcutaneous tissue and fascia [1] with mortality from $6 \%$ to $80 \%$ [2] . The infection travels along planes and later can potentially involve deeper muscles with resultant myositis and myonecrosis.

The etiology is based on mono or polymicrobial infection and most frequently arises from odontogenic infections [3]. Nowadays the management is based on a rapid and aggressive surgical treatment with removal of the necrotic tissue along with broad spectrum antibiotic therapy. Some facilities have also included hyperbaric oxygen therapy (HBOT) [4]. The Italian Society of Undersea and Hyperbaric Medicine (SIMSI) is withheld the Italian authority on the indications for HBOT. In the 2nd edition of their guidelines HBOT was also recommended as adjuvant therapy in NSTIs [5]. Despite this, HBOT is not usually used as a standard of care in these patients and this happens for several reasons: not all hospitals are equipped with a hyperbaric medicine service, a high percentage of doctors do not know the usefulness of HBOT and its possible use in these conditions and well-controlled, randomized, clinical trials demonstrating a statistically significant benefit of HBOT are lacking and consequently its use as an adjunctive therapy for NSTIs remains controversial[6]. Besides transfer to a hospital equipped with HBOT, we should never delay emergency surgical intervention.

We report the case of a young patient affected by NSTI resulting from dental infection and complicated by necrotizing myositis of right pectoral muscle treated with standard of care plus HBOT as adjuvant therapy.

\section{Case report}

A 37 years old man was admitted to our maxillo facial surgery department with a two weeks history of dental pain under treatment with antibiotic therapy at home. During this time, after extraction of the lower right third molar, there was a progressive swelling of the submandibular and laterocervical region showing tense and painful skin and progressive involvement of the right upper thoracic region. The patient did not report any pathologies in his past medical history. On admission he was conscious and cooperating, his body temperature was $37,5^{\circ} \mathrm{C}$, blood pressure $110 / 60 \mathrm{mmHg}$, heart rate 94 b.p.m., oxygen saturation on room air 
98\% with no signs of respiratory exertion. Physical examination revealed swelling of the latero-cervical region with involvement of the right pectoral region where the skin appeared tense, burning and painful on palpation (fig 1). Our examination of the oral cavity revealed a poor oral hygiene condition with inflammation of the gingival mucosa and leak of purulent material in the gingival fornix . The computed tomography (CT) scan of the head, neck and thorax with contrast was performed and revealed locules of gas and ill-defined low attenuation in the cervical area and in the thigh chest muscle, highly suspicious for a gas-forming infection (figure 2-3).

Routine laboratory report as follow: white blood cell count $15.230 \mathrm{~mm} 3, \mathrm{Hb}$ 14,5 gr/dl, CPK 414 U/L, CRP $150 \mathrm{mg} / \mathrm{L}, \mathrm{PCT}$ 0.94. The day after admission the pain and swelling increased and he developed dysaesthesia on the right upper thoracic zone. A compartment syndrome due to infection was diagnosed, thus patient was urgently carried on operating room where underwent to extensive surgical debridement of right pectoral area. The operating field showed interstitial "dishwater-like" fluid and discolored poor contractile muscles, with pockets of necrosis and pus. The tissues were irrigated with $10 \mathrm{~L}$ of saline and based on advice with infectious disease empiric antibiotic therapy was started with piperacilline-tazobactam $4,5 \mathrm{gr}$ ev/4 die and daptomycin $8 \mathrm{mg} / \mathrm{kg} /$ day ev. Blood cultures were performed pre-operatively with a sterile peripheral vein technique and during surgery cultures were taken from the surgical site. After surgery patient was extubated and the day after he started HBOT as adjunctive therapy in the treatment of NSTIs and myositis. The HBOT treatment consisted in two dives with $100 \%$ oxygen in a pressure chamber 2,8 atmospheres absolute within the first two days, and then daily for a total of 16 session. Clinical improvement was observed after third day of HBOT and three weeks after admission patient was discharged at home in good health (figure $4)$.

\section{Discussion}

NSTIs are infectious diseases burdened with high mortality that spreads through the fascial planes and leads to connective tissue necrosis. NSTIs may occur as a complication of a variety of surgical procedures or medical conditions and it may also be idiopathic, as in scrotal or penile necrotizing fasciitis. The prevalence is higher in males and in patients aged 45 to 64 years. Physical findings may not be commensurate with the degree of patient discomfort. Early in the disease course, the patient may look misleading well; unfortunately, this may interfere with early detection, which is the cornerstone to a favorable outcome. As reported by our previous work[7], the sites from which NSTIs may originate in the cervical district are represented in order of frequency by:

odontogenic infection, peritonsillar abscess and sinusitis.

According to the depth of tissue infection and necrosis, NSTs can be classified into three forms which involve the dermis and subcutaneous tissue for necrotizing cellulitis, the fascia for necrotizing fasciitis, and the muscle layer with the intact overlying skin for necrotizing myositis [8] .

Cervical fascia is the fibrous tissue that divides structures of the neck and is divided into superficial and deep layers. The superficial layer of the deep cervical fascia envelops parotid and submandibular glands and it is the external border of the odontogenic infections of the neck [9]. Most of these infections originate from the second or third mandibular teeth infection or extraction. The roots of the second and third molars can extend up to the submandibular space and through this path the infections of this site can spread through the cervical fascia to the lateral pharyngeal space, involve the retropharyngeal space and track into the anterior or to the posterior mediastinal region with the clinical picture of mediastinitis [10]. The infection can be monomicrobial or polymicrobial. The most frequently isolated organisms in NSTI include viridans group streptococci, S. aureus, peptostreptococci, Bacteroides spp., Prevotella spp. and Fusobacterium[11]

Antibiotic therapy must be started early, must be empirical and wide spectrum in the early stages, subsequently tailored on the basis of the results obtained from cultural tests performed on biological material. Furthermore, in the choice of drugs used, other variables should be carefully considered, such as the risk factors for multidrug-resistant microorganisms. Finally, a further key point consists is the rapid antibiotic steward ship which must be based on clinical evidence and bio-humoral markers. Serum procalcitonin has 
been proposed as a biomarker capable of guiding the response to antibiotic therapy, allowing a rapid deescalation. Normal serum procalcitonin concentration in a healthy individual is $<0.05 \mathrm{ng} / \mathrm{mL}$. Patients with procalcitonin concentration of $<0.5 \mathrm{ng} / \mathrm{mL}$ are unlikely to have sepsis. However, this concentration does not exclude infection, because localized infections like cervical NSTI without systemic signs are still possible[12] . Regrettably, with high frequency, antibiotic therapy is started on an empirical basis without providing for targeted culture samples and subsequently followed superficially.

Cervical NSTIs uncommonly gets complicated with the involvement of muscles but when that happens, as happened in our patient, a theatre of necrotizing myositis occurs. Necrotizing myositis is a surgical emergency. It may presents without changes in the skin thus it is under appreciated and diagnosis is often delayed [13] . In our patient the entry route was through dental infection and this is in accordance with a literature review published by Pellis et al [14], wich highlighted how the teeth are the main (65\%) responsible cause of NSTIs of the cervical region. In our case infection, luckily did not involve the deep cervical fascial planes, but spread along the superficial planes and affect the subcutaneous skin and muscles of the right upper thoracic region, as evidenced by the images.

HBOT has been proposed as an adjunctive therapy for patients with NSTIs[15] . In HBOT, patients breathes $100 \%$ oxygen for a specified period of time in a pressurized chamber at pressure higher than atmospheric pressure at sea level. It causes increased blood and oxygen content in hypoxic tissues and this increased oxygen availability is responsible for multiple effects as antimicrobial activity on both aerobic and anaerobic bacteria thereby demonstrating the bactericidal and bacteriostatic effects due to enhanced mobility and bacteriophagic activity of leukocytes. Parts of the antimicrobial effects of HBOT are believed to result of both formation of reactive oxygen species (ROS)[16] and synergistic effects with some antibiotics [17]

On top HBOT promotes granulation tissue formation due to fibroblast proliferation and collagen synthesis, and improves microcirculation due to edema reduction[18] . The increase in $\mathrm{O} 2$ tension produced by HBOT, which persists for several hours after therapy, is responsible for angiogenic properties of HBOT [19] Hyperbaric $\mathrm{O} 2$ causes reduced platelet aggregation, improved tissue microcirculation, and diminished metabolic disturbances. These properties along with increased dissolved $\mathrm{O} 2$ in plasma lead to better oxygenation of hypoxic tissue, where red blood cells cannot reach.[20]

The hyperbaric treatment of our patient was conducted on the basis of the SIMSI guidelines [5]: in the first 3 days, we adopted the maximum oxygen dosage at 2.8 ATA $(284 \mathrm{kPa})$, twice day. From the 4th day: treatments become daily with sessions at 2.5 ATA $(253 \mathrm{kPa})$. The duration of each session was 80 minutes and the treatment was continued until the infection is considered to be overcome.

Absolute contraindications to HBOT are represented by non-drained pneumothorax, $\mathrm{PaO} 2$ / FiO2 ratio $<200$ assessed by blood gas analysis, seizures, claustrophobia and psychotic pictures in rupture. Our patient had none of these, he showed good compliance with the method with a good response to treatment from the third day of HBOT.

\section{Conclusion}

HBOT should be added to surgical treatment and antibiotics for all NSTIs patients if there is no contraindications. Prompt HBOT could be useful for preventing mortal complications and for reducing costs, associated with the management of these patients, through a shorter length of stay and a reduction in relapses. 